\title{
Low-Frequency Capacitive Sensing for Environmental Monitoring of Water Pollution with Residual Antibiotics
}

\author{
M. Soprani ${ }^{1,2}$, O. Korostynska ${ }^{1}$, A. Mason ${ }^{1}$, \\ A. Amirthalingam ${ }^{1}$, J. Cullen $^{1}$, M. Muradov ${ }^{1}$, \\ A. Al-Shamma'a ${ }^{1}$ \\ ${ }^{1}$ Faculty of Engineering and Technology \\ Built Environment and Sustainable Technologies \\ Research Institute \\ Liverpool John Moores University \\ Liverpool, United Kingdom \\ o.korostynska@,ljmu.ac.uk
}

\author{
V. Sberveglieri ${ }^{3}$, E. N. Carmona ${ }^{3,4}$, \\ G. Sberveglieri ${ }^{3,4}$ \\ ${ }^{2}$ Department of Life Science \\ University of Modena and Reggio Emilia \\ Modena, Italy \\ ${ }^{3}$ CNR-INO SENSOR lab \\ Brescia, Italy \\ ${ }^{4}$ Department of Information Engineering \\ University of Brescia \\ Brescia, Italy
}

\begin{abstract}
The intensive use of antibiotics for human treatment and disease prevention in livestock and agriculture has led to the global threat of antibiotic resistance. To prevent the contamination of antibiotic residues through wastewater treatment plants, this paper assesses the feasibility of using UV-Vis spectrophotometer and lowfrequency impedance analysis for detecting specific antibiotics in water. The change in the optical absorbance recorded with the spectrophotometer and the change in the values of capacitance recorded using impedance analyzer were dependent on the type of antibiotic and its concentration in water. The efficiency of the sensing methods in detecting different antibiotics at different concentrations is analyzed throughout the paper.
\end{abstract}

Keywords- Capacitance sensing system; UV-Vis spectroscopy; antibiotics detection; water quality monitoring; pollution prevention.

\section{INTRODUCTION}

Antibiotics are drugs used by human, animals and plants for the purposes of treating bacterial infections [1]. The application of antibiotics has been increasing exponentially; the global human consumption of antibiotics has risen by $30 \%$ between 2000 and 2010 [2] whereas in 2010, at least 63,200 tons of antibiotics were consumed by livestock. By 2030 , this figure is projected to rise 105,600 tons, to meet the demands of a projected 8.5 billion human population [2]. Increased use of antibiotics causes health and environmental risks of antibiotic resistance and more than two million people are affected with antibiotic-resistant infections every year.

When the bacteria are exposed to antibiotics continuously, they develop immunity to them, they become adapted to the antibiotic environment and the strongest bacteria survive and continue to multiply in the presence of antibiotics [1].
Wastewater treatment plants are identified as one of the important source of antibiotic contamination of surface water [3]. Considerable attention has been paid to pharmaceutical antibiotics and several methods have been proposed to detect pharmaceutical antibiotics and antibioticresistant bacteria in wastewater treatment process. However, little attention has been drawn towards antibiotic use in farm animals and the contribution of wastewater treatment plants to antibiotic resistance.

This paper reports on the results of a proof-of-concept study in an attempt to provide a sensing system for real-time monitoring of water pollution with antibiotics. For this purpose, two different animal antibiotics, namely Lincomycin and Tylosin (Fig. 1 a,b) (Fig. 2 a,b) were chosen, and their presence and concentration were detected by UV-Vis spectrophotometer and impedance analyzer. As Fig. 1 show, these solutions are transparent and contaminated water user will not be even aware of the antibiotics presence, until it could be too late. Therefore, novel real-time antibiotics sensing techniques are required.

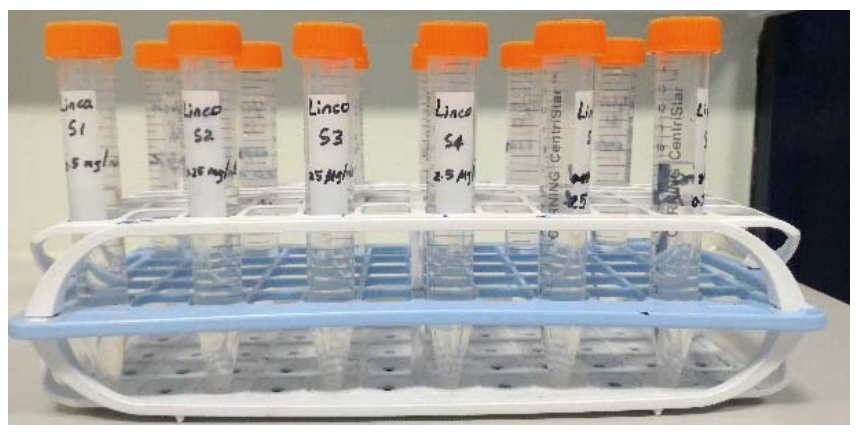

Fig 1a Different concentrated solutions of Lincomycin. 


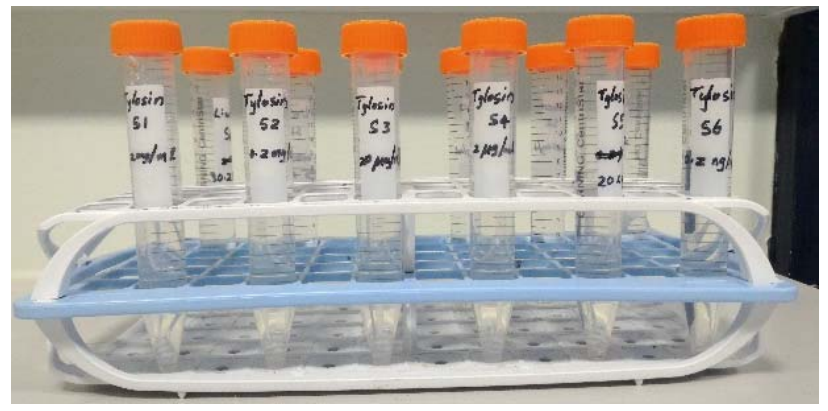

Fig 1b Different concentrated solutions of Tylosin.

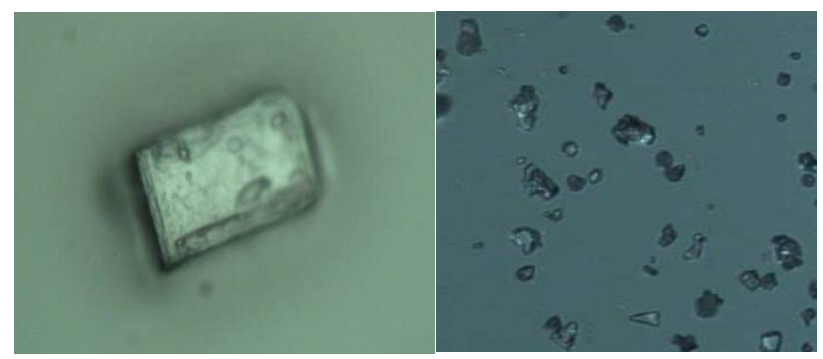

Fig. 2a,b Optical image of Lincomycin and Tylosin particles taken with ZEISS Scope.A1 20x/0.4 EC-EPIPLAN microscope.

Tylosin $\left(\mathrm{C}_{46} \mathrm{H}_{77} \mathrm{NO}_{17}\right)$ is a fermentation product of Streptomyces fradiae and it is used against gram-positive bacteria and some of the gram-negative bacteria $[4,5]$.

Lincomycin $\left(\mathrm{C}_{18} \mathrm{H}_{34} \mathrm{~N}_{2} \mathrm{O}_{6} \mathrm{~S}\right)$ comes from the actinomyces Streptomyces lincolnensis and it is also used to treat grampositive bacterial infections in food animals $[6,7]$. However, Lincomycin is used to treat severe bacterial infections in people who cannot receive penicillin antibiotics. When the bacteria are exposed to Lincomycin through antibiotic contaminated animal food, water and soil, they become resistant to it, which leads to inactivation deactivation of Lincomycin in both human and animals.

When antibiotic-resistant bacteria develop in industrial livestock, they can reach human population through food, water, soil and air. In particular, antibiotics have become chemicals of emerging concern to the public because of their potential to reach drinking-water.

Antibiotic resistant bacteria can cause pneumonia, toxic shock, skin abscesses, heart valve infections and serious medical conditions in humans [8]. Depending on circumstances [9], a range of methods is currently used to detect the presence and type of antibiotics in various media. The following section provides critical overview of modern methods that are used to monitor antibiotics in wastewater.

\section{MODERN Methods OF ANTIBIOTICS DETECTION IN WASTEWATER}

Trace concentrations of antibiotics in wastewater, various water sources and some drinking-waters can be currently found worldwide. Concentrations in surface waters and groundwater were $3.6 \times 10^{-4} \mu \mathrm{g} / \mathrm{ml}$ of Lincomycin and $1.5 \times 10^{-3} \mu \mathrm{g} / \mathrm{ml}$ of Tylosin [10], whereas concentrations in treated water were generally below $5 \times 10^{-5} \mu \mathrm{g} / \mathrm{ml}$ (or 50 $\mathrm{ng} / \mathrm{l})[11]$.
Due to the low concentration of pharmaceuticals in water, in order to detect them, tools and techniques of analysis will have to be more sensitive.

In current methods of detecting antibiotics in wastewater, high performance liquid chromatography (HPLC) and tandem mass spectrometry (MS/MS) were used after the extraction process. For example, in an approach to detect occurrence of several antibiotics in hospital, residential, dairy and municipal wastewater using HPLC and MS/MS, Lincomycin was detected with some other antibiotics in hospital and dairy wastewater [12].

Ultra-high performance liquid chromatography-tandem mass spectrometry (UPLC-MS/MS) method was developed to detect selected pharmaceutical antibiotics in wastewater and the method showed sensitive, reliable and accurate performance to three antibiotics [13].

A molecular biological method of PCR was used to find antibiotic resistant bacteria in wastewater biofilms and also in drinking water biofilms [14]. PCR detected Vancomycinresistant enterococci in wastewater and in drinking water and also detected methicillin-resistance staphylococci in hospital wastewater. In another instance, the spread of antibiotic resistance genes in wastewater treatment systems and their occurrence in surface and drinking water systems were revealed by quantitative PCR (qPCR) assay [15].

On the development of sensors for detecting a specific antibiotic in wastewater effluents as well as in natural water, an electrochemical sensor in a form of a porous nafion multi-walled carbon nanotube composite film electrode, an electrochemical sensor, was developed to detect the antibiotic Ciprofloxacin (CFX) [16]. The sensor was selective only for Ciprofloxacin even with the presence of other antibiotics and non-target water constituents [16]. Therefore, it was recommended to be applicable in wastewater effluents and in natural waters to detect Ciprofloxacin.

HPLC method was compared with enzyme-linked immunosorbent assay (ELISA) for detection of Tylosin and Tylosin-related compounds in water [17]. As a result, HPLC detected a lower concentration Tylosin compound while ELISA detected most of the Tylosin-related compounds.

The combination of ELISA with HPLC/MS or with other conventional detection methods was suggested to be potential for sensitive and specific detection of Tylosinrelated compounds. HPLC with MS/MS is the commonly and widely used technology in detecting antibiotics in wastewater as well as in surface water. However, this method is too expensive and time-consuming for real-time water pollution monitoring at multiple locations. This research aims to address this issue by assessing the capabilities of UV-Vis Spectrophotometer and Impedance analyzer to detect the presence, type and concentration of Lincomycin and Tylosin in water in real time. 


\section{EXPERIMENTAL PROCEDURE}

Antibiotic solutions of Lincomycin and Tylosin were prepared by dissolving $25 \times 10^{3} \mu \mathrm{g}$ and $20 \times 10^{3} \mu \mathrm{g}$ of respective antibiotics in $10 \mathrm{ml}$ of deionized water to achieve the final concentrations of $2.5 \times 10^{3} \mu \mathrm{g} / \mathrm{ml}$ and $2.0 \times 10^{3} \mu \mathrm{g} / \mathrm{ml}$ respectively. Six different concentrated antibiotic solutions were prepared in each case, from $2.5 \times 10^{3} \mu \mathrm{g} / \mathrm{ml}$ to $2.5 \times 10^{-4}$ $\mu \mathrm{g} / \mathrm{ml}$ of Lincomycin and from $2.0 \times 10^{3} \mu \mathrm{g} / \mathrm{ml}$ to $2.0 \times 10^{-4}$ $\mu \mathrm{g} / \mathrm{ml}$ of Tylosin, by appropriate dilution of the respective antibiotic solution with deionized water.

To assess the feasibility of using capacitance sensing approach for residual antibiotics concentration monitoring, a comprehensive set of experiments was conducted. A capacitor sensing system connected to a Hameg Industries Programmable LCR Bridge HM8118 was used, as illustrated in Fig. 3.

In parallel, UV-Vis spectroscopy was employed for the optical measurements in $200-1000 \mathrm{~nm}$ wavelength range using Jenway 7315 Spectrophotometer.

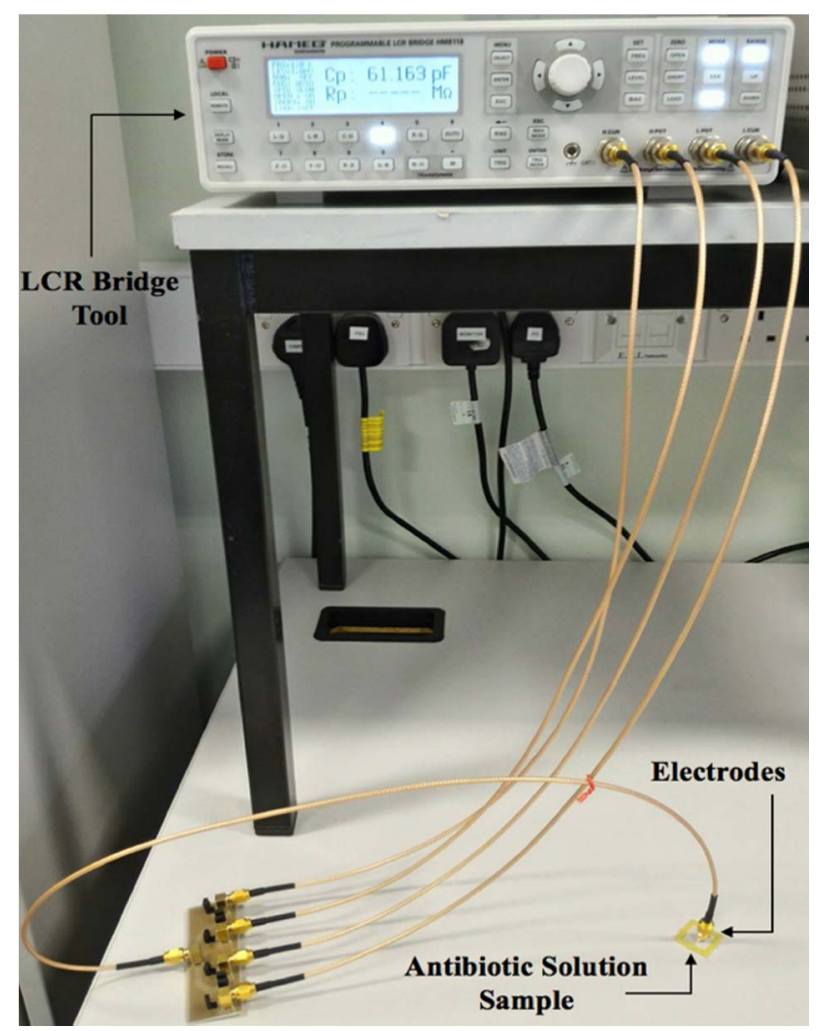

Fig. 3 Capacitance Measurement of Antibiotics Solutions.

In this experiment, two cylindrical rods of a typical SMA connector were used as the electrodes for capacitance measurements in $20 \mathrm{~Hz}-200 \mathrm{kHz}$ frequency range $[18,19]$. The electrical field is formed between the two electrodes through, the voltage applied across them (Fig. 4).

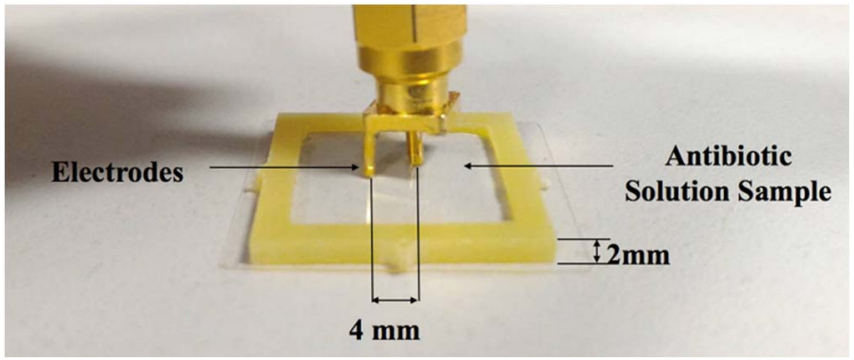

Fig. 4 Capacitor composed of two cylindrical rods electrodes.

The capacitance describes the effects on the electric field due to the space between the two plates and it is determined by the geometry of the conductors, the distance between the electrodes and the dielectric material [20]. Value of capacitance can be found according to the following equation:

$$
C=\frac{\pi \varepsilon_{0} \varepsilon_{r}}{\ln \frac{d}{r}} L
$$

where $C$ is the capacitance in farads (F),

$\varepsilon_{0}$ is the relative static permittivity (dielectric constant) of the material between the plates,

$\varepsilon_{r}$ is the permittivity of free space, which is equal to $8.854 \mathrm{x}$ $10^{-12} \mathrm{~F} / \mathrm{m}$,

$L$ is the rod length in meters,

$d$ is the separation distance (in meters) of the two rods, $r$ is the radius of the rod in meters.

A capacitive sensor uses the changes in the value of the dielectric constant, manifested as a change in electrical signal, as an indicator of water pollution, in this case with various antibiotics [21]. Since the geometry of conductor and the distance between the electrodes are constant (Fig. 4) the dielectric constant represents the only variable [21]. That means a capacitance change depending on the different solutions concentrations.

The experiments provide a capacitance measurement for the antibiotics solutions at different concentrations, and were completed in regulated temperature and humidity conditions. The frequency range used for the measurement of capacitance in the antibiotics solutions was from $20 \mathrm{~Hz}$ to $200 \mathrm{kHz}$, they are generated from an LCR Bridge tool, which was connected to a desktop computer for data acquisition via a bespoke LabVIEW interface. The measurements were taken three times for each sample with 3 -second interval and then averaged.

In order to do the analysis $400 \mu 1$ of solution were put inside the sample holder, then the electrodes were immersed inside the solution samples and finally, the capacitance values were repeatedly measured for the whole range of frequencies. The graphs in the next section present averaged results, with max $5 \%$ variations between the samples. 


\section{RESUlTS AND DISCUSSION}

The maximum of absorbance for the antibiotics was observed in the range between $200 \mathrm{~nm}$ and $400 \mathrm{~nm}$ as shown in Fig. 5 a,b.

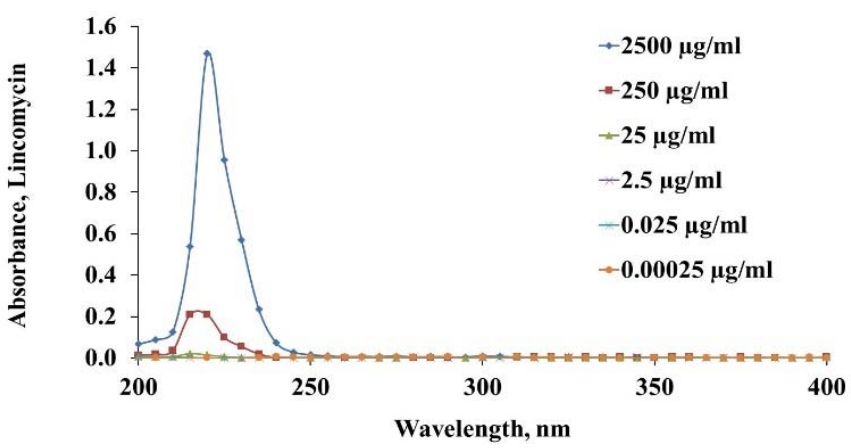

Fig. 5a Maximum absorbance of Lincomycin in the range between $200 \mathrm{~nm}$ to $400 \mathrm{~nm}$.

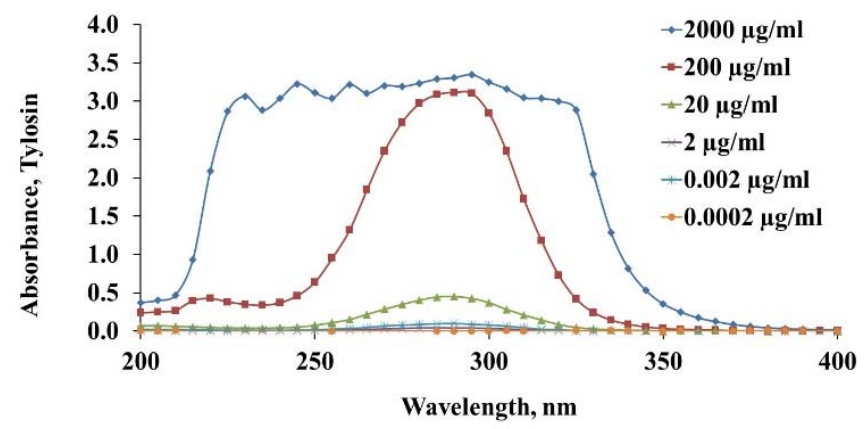

Fig. 5b Maximum absorbance of Tylosin in the range between $200 \mathrm{~nm}$ to $400 \mathrm{~nm}$

In particular, the change in the optical absorption in UVVis range with increasing concentration of Lincomycin and Tylosin is depicted in Fig. 6 and Fig. 7. This set of measurements served as a benchmark for validating concentrations of antibiotics and for comparison of sensitivities of standard optical and novel capacitance sensing approaches. Fig. 6 and Fig. 7 illustrate the change in the optical absorption with concentration at $220 \mathrm{~nm}$ wavelength for Lincomycin and $290 \mathrm{~nm}$ wavelength for Tylosin. With UV-Vis, the type of antibiotic and its concentration can be identified at high concentration and also at a specific wavelength. There is not any positive absorbance observed at low concentrations. Therefore, it is not possible to distinguish between these two antibiotics at low concentrations in the same solution.

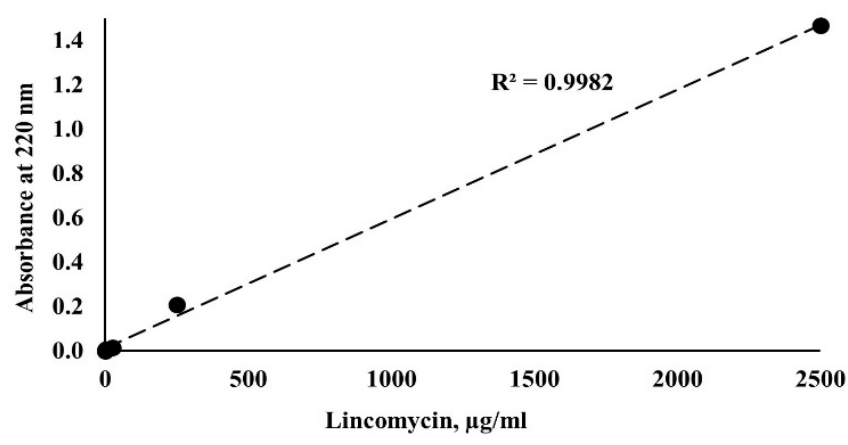

Fig. 6 Absorbance change with Lincomycin concentration at $220 \mathrm{~nm}$.

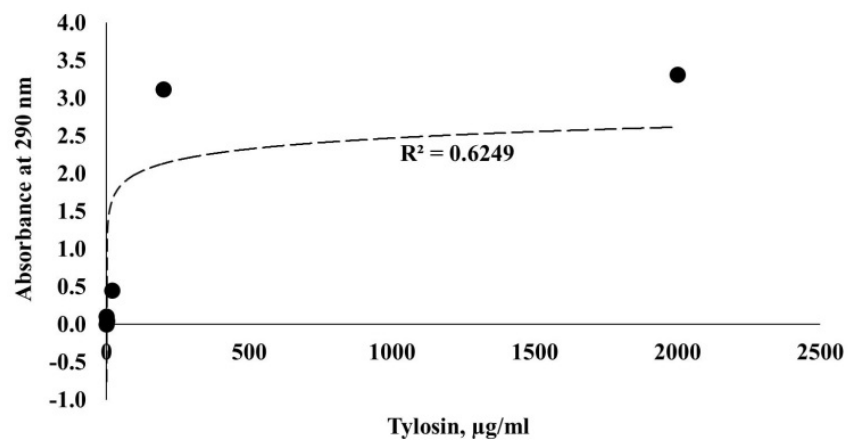

Fig. 7 Absorbance change with Tylosin concentration at $290 \mathrm{~nm}$.

To assess the capabilities of the capacitance sensing system for monitoring concentration of Lincomycin and Tylosin antibiotics in the solution, the system shown in Fig. 3 was used.

Notably, all the measurements were repeated numerous times, and the graphs represent the averaged values. The change in the sensor response caused by the presence of antibiotics in various concentrations was higher than a change possibly caused by a noise.

From the low frequencies range used, the major discrimination between the samples was at $50 \mathrm{~Hz}, 100 \mathrm{~Hz}$ and $500 \mathrm{~Hz}$. The linear dependences of capacitance with respect to Lincomycin and Tylosin concentration at $50 \mathrm{~Hz}$, $100 \mathrm{~Hz}$ and $500 \mathrm{~Hz}$ recorded for $400 \mu \mathrm{l}$ solutions placed into at the sample folder are shown respectively in Fig. 8 and Fig. 9.

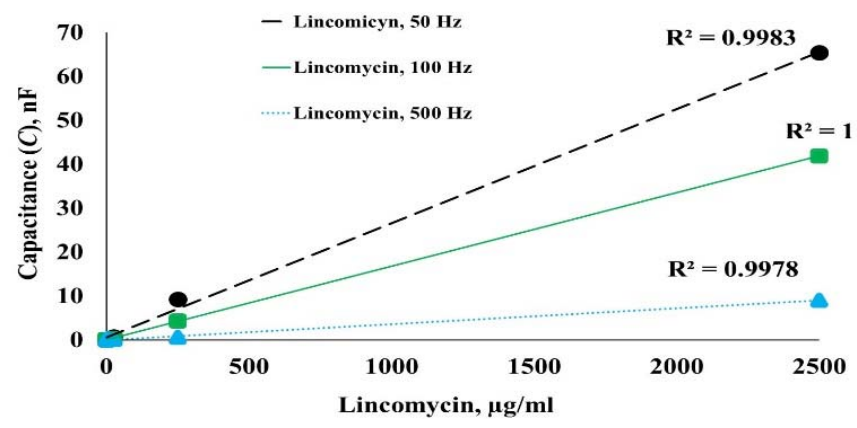

Fig. 8 Capacitance change with Lincomycin at $50 \mathrm{~Hz}, 100 \mathrm{~Hz}$ and $500 \mathrm{~Hz}$. 


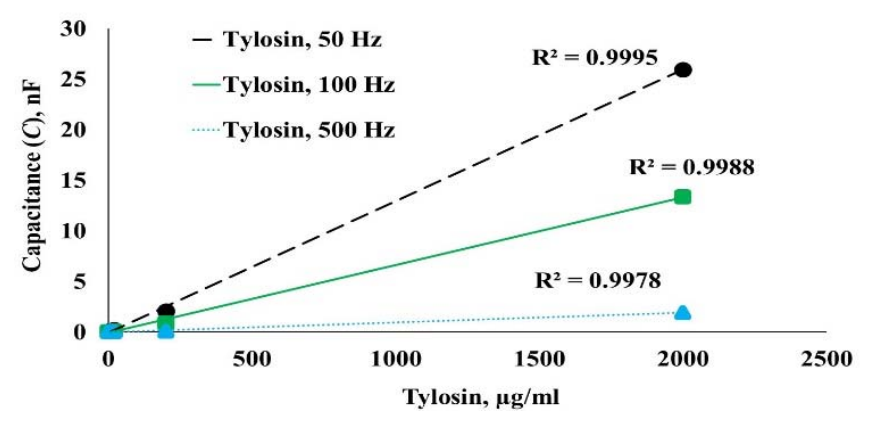

Fig. 9 Capacitance change with Tylosin at $50 \mathrm{~Hz}, 100 \mathrm{~Hz}$, and $500 \mathrm{~Hz}$.

The high linear dependence of the capacitance values on the concentration measured for both types of antibiotic solutions can be used as a basis for the capacitance sensing system, which would be able to quantify in real time the concentration of different types of antibiotics present in aqueous media. Fig. $10 \mathrm{a}, \mathrm{b}$ and $\mathrm{c}$ demonstrate that at selected frequencies of $50 \mathrm{~Hz}, 100 \mathrm{~Hz}$, and $500 \mathrm{~Hz}$, respectively, the sensing platform was able to distinguish between the two antibiotics in a full range of concentrations. Note that although the trend in the signal behaviour is similar, the measured capacitance value is different for each antibiotic and fixed frequencies and these present a unique "fingerprint response pattern" that can be used for computer signal processing.

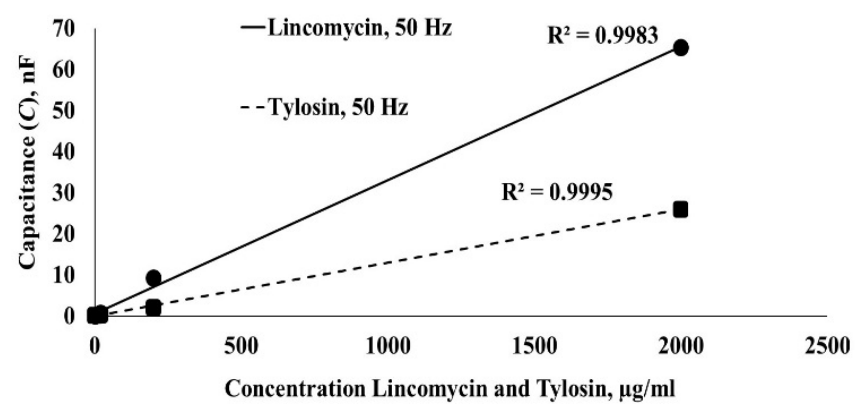

Fig. 10a Change in measured capacitance at $50 \mathrm{~Hz}$ with increasing Lincomycin and Tylosin concentration.

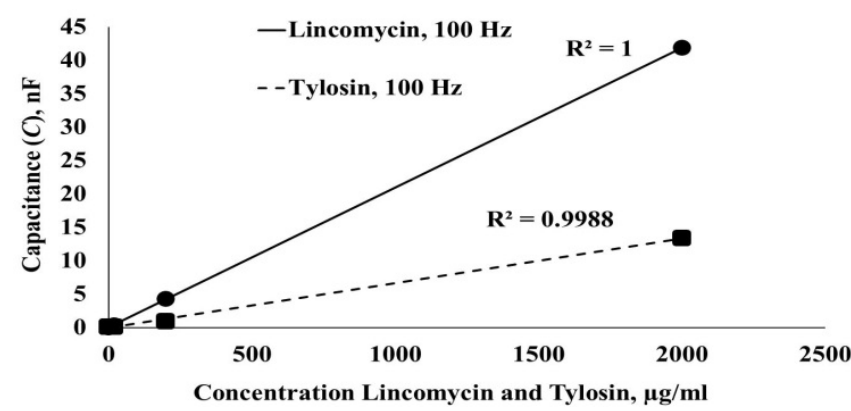

Fig. 10b Change in measured capacitance at $100 \mathrm{~Hz}$ with increasing Lincomycin and Tylosin concentration.

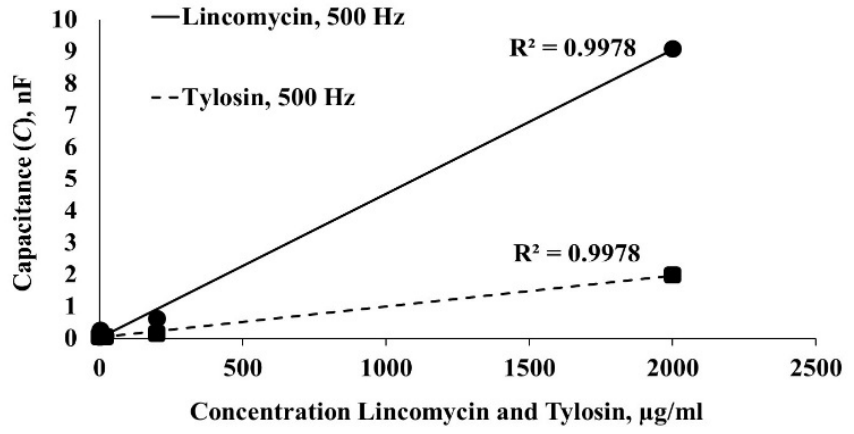

Fig. 10c Change in measured capacitance at $500 \mathrm{HZ}$ with increasing Lincomycin and Tylosin concentration.

To illustrate that the proposed capacitive sensing approach is feasible even at the lowest concentrations of antibiotics present in water solution, Fig. $10 \mathrm{~d}$ depicts the change in the value of capacitance with Lincomycin and Tylosin concentration in $0-20 \mu \mathrm{g} / \mathrm{ml}$ range.

Although the trend in the graphs obtained is similar for the two antibiotics, the absolute values of capacitances are specific to each antibiotic and when a number of frequencies used for analysis, these responses form unique pattern, fingerprint signature response of each antibiotic. Thus, suggested capacitive sensing approach can provide both qualitative and quantitative characterisations of water samples in real time.

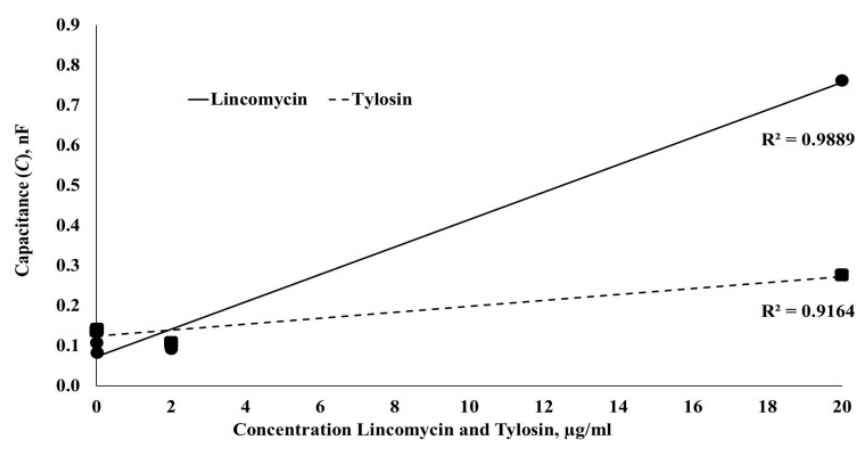

Fig. 10d Change in capacitance value of Lincomycin and Tylosin solutions in $0-20 \mu \mathrm{g} / \mathrm{ml}$ range measured at $50 \mathrm{~Hz}$.

Importantly, it was experimentally confirmed that the capacitance sensing system is capable of differentiating between the type of antibiotics present in water, as well as to measure their concentration at $20 \mathrm{~Hz}-200 \mathrm{kHz}$ frequency range. However, selecting only three different sampling frequencies of $50 \mathrm{~Hz}, 100 \mathrm{~Hz}$ and $500 \mathrm{~Hz}$ was enough to clearly differentiate between the samples. The results obtained allow building a novel device formed by an array of sensors working at different selective low frequencies for Lincomycin and Tylosin. That means the possibility to analyze those antibiotics inside a complex sample with other antibiotic types.

In particular, the sensing system is able to detect a residual antibiotics concentration in water up to $2.0 \times 10^{-4}$ $\mu \mathrm{g} / \mathrm{ml}$, which is below the concentrations of Lincomycin and Tylosin found in wastewater. 
For drinking water, the permitted concentration of these antibiotics is less than $5 \times 10^{-5} \mu \mathrm{g} / \mathrm{ml}$. Therefore, further research to improve the sensitivity of the sensors using different technologies is ongoing, including developing novel electromagnetic waves sensor to address the need of water quality monitoring in real time.

\section{CONCLUSION}

This paper communicates the experimental results of using a capacitance sensing system for the determination of antibiotic concentrations and type, in particular Lincomycin and Tylosin. A comprehensive set of complementary experiments using optical and capacitance detection methods confirmed the potential of this novel sensing approach to serve as an alternative method of residual antibiotics concentration and type monitoring in a wide range of applications, including food industry and environmental monitoring. In particular, the presented sensor concept demonstrates relevance for wastewater monitoring, as its sensitivity is within the range allowable for wastewater. However, the requirements for the safety of drinking water are far more stringent, and therefore further developments are necessary. The team is working on a bespoke electromagnetic wave sensor to address this issue.

Importantly, once the frequency range where the system is selectively sensitive to a particular antibiotic is known, there is no need to use all spectrum range and so, the expensive capacitance equipment could be replaced with custom-made hand-held electronics units for portable applications, where the measurements can take place anywhere and provide instant results.

\section{REFERENCES}

[1] F. Baquero, J.-L. Martinez, and R. Canton, "Antibiotics and antibiotic resistance in water environments," Current Opinion in Biotechnology, vol. 19 , pp. $260-265,2008$.

[2] E. P. The Center for Disease Dynamics, "The State of the World's Antibiotics 2015," 2015.

[3] A. L. Batt, S. Kim, and D. S. Aga, "Comparison of the occurrence of antibiotics in four full-scale wastewater treatment plants with varying designs and operations," Chemosphere, vol. 68, pp. 428-435, 2007.

[4] P. P. Gray, "TYLOSIN." vol. 3, ed: Pergamon Press, 1985, pp. 83-93.

[5] S. Khaliq, N. Rashid, K. Akhtar, and M. A. Ghauri, "Production of tylosin in solid-state fermentation by Streptomyces fradiae NRRL2702 and its gamma-irradiated mutant (-1)," Letters in Applied Microbiology, vol. 49, pp. 635-640, 2009.
[6] J. E. Gonzalez and T. L. Miller, "LINCOMYCIN." vol. 3, ed: Pergamon Press, 1985, pp. 211-223.

[7] M. Koběrská, J. Kopecký, J. Olšovská, M. Jelínková, D. Ulanova, P. Man, et al., "Sequence analysis and heterologous expression of the lincomycin biosynthetic cluster of the type strain Streptomyces lincolnensis ATCC 25466," Folia Microbiologica, vol. 53, pp. 395 401, 2008.

[8] K. Kümmerer, "Antibiotics in the aquatic environment - A review Part I," Chemosphere, vol. 75, pp. 417-434, 4// 2009.

[9] O. Korostynska, A. Mason, A. Al-Shamma'a, W. Jansomboon, and S Boontanon, "Real-time detection of residual antibiotics concentration with microwave cavity and planar EM sensors," in 2015 9th International Conference on Sensing Technology (ICST), 8-10 Dec. 2015, Piscataway, NJ, USA, 2015, pp. 492-5.

[10] K. G. Karthikeyan and M. T. Meyer, "Occurrence of antibiotics in wastewater treatment facilities in Wisconsin, USA," Science of the Total Environment, vol. 361, pp. 196-207, 2006.

[11] W. H. Oragization, "Pharmaceuticals in drinking-water," 2012.

[12] K. D. Brown, J. Kulis, B. Thomson, T. H. Chapman, and D. B. Mawhinney, "Occurrence of antibiotics in hospital, residential, and dairy effluent, municipal wastewater, and the Rio Grande in New Mexico," Science of the Total Environment, vol. 366, pp. 772-783, 2006.

[13] P. Qiu, X. Guo, N. Wang, X. Kong, and H. He, "[Simultaneous determination of ten antibiotics in pharmaceutical wastewater using ultra-high performance liquid chromatography-tandem mass spectrometry]," Se $\quad p u=$ Chinese journal of chromatography/Zhongguo hua xue hui, vol. 33, pp. 722-729, 2015.

[14] T. Schwartz, W. Kohnen, B. Jansen, and U. Obst, "Detection of antibiotic-resistant bacteria and their resistance genes in wastewater, surface water, and drinking water biofilms," FEMS Microbiology Ecology, vol. 43, pp. 325-335, 2003.

[15] A. Lupo, S. Coyne, and T. U. Berendonk, "Origin and evolution of antibiotic resistance: the common mechanisms of emergence and spread in water bodies," Analyzing possible intersections in the resistome among human, animal and environment matrices, p. 116, 2012.

[16] P. Gayen and B. P. Chaplin, "Selective Electrochemical Detection of Ciprofloxacin with a Porous Nafion/Multiwalled Carbon Nanotube Composite Film Electrode," ACS Applied Materials and Interfaces, vol. 8, pp. 1615-1626, 2016.

[17] D. Hu, B. Fulton, K. Henderson, and J. Coats, "Identification of tylosin photoreaction products and comparison of ELISA and HPLC methods for their detection in water," Environmental Science and Technology, vol. 42, pp. 2982-2987, 2008.

[18] E. Terzic, R. Nagarajah, and M. Alamgir, "A neural network approach to fluid quantity measurement in dynamic environments," Mechatronics, vol. 21, pp. 145-155, 2// 2011.

[19] H. Kuttruff, Ultrasonics: Fundamentals and applications: Springer Science \& Business Media, 2012.

[20] W. Bolton, "Capacitance," Engineering Science 2006.

[21] R. A. J. J. W. Serway, "Capacitance and dielectrics," Physics for scientists and engineers, vol. 6, pp. 796-820, 2004. 